\title{
Hiperaldosteronismo primário - uma causa de hipertensão arterial
}

Fátima Carvalho Matos*

\section{RESUMO}

Enquadramento: A grande maioria dos doentes com hipertensão arterial apresenta a forma primária. No entanto, podem surgir formas secundárias no decorrer da doença, sendo a mais prevalente o hiperaldosteronismo primário. Entre as duas principais etiologias encontram-se o adenoma produtor de aldosterona e a hiperplasia supra-renal, sendo a primeira mais comum. Descrição do caso: Doente de 74 anos, hipertenso, com fibrilhação auricular crónica, controlado com diuréticos e anti-arrítmico, submetido a valvuloplastia mitral por estenose, apresenta-se na consulta por elevação dos níveis tensionais, associada a palpitações e síncope. Na avaliação laboratorial foi detectada hipocaliemia, o que levantou a suspeita de efeito adverso de diurético e/ou de hiperaldosteronismo. O último diagnóstico foi confirmado após estudos complementares, que indicaram aldosterona plasmática e urinária elevadas e renina plasmática baixa. A tomografia computorizada detectou nódulo sólido na supra-renal direita, compatível com adenoma. O doente foi referenciado a consulta de Endocrinologia, medicado com espironolactona. Aguarda eventual adrenalectomia unilateral.

Comentário: O caso clínico evidencia que, durante a evolução da hipertensão arterial, podem surgir formas secundárias, potencialmente curáveis, para as quais o médico de família deve estar alerta. Procedendo-se a uma história clínica detalhada e a um exame físico rigoroso, associados ao pedido descriminado de exames complementares de diagnóstico, é possível conhecer a etiologia da hipertensão arterial secundária. Este caso é ilustrativo da relevância que tem a continuidade de cuidados prestados pelo médico de família e a boa articulação entre os cuidados primários e os cuidados hospitalares.

Palavras-chave: Hipertensão Secundária; Hiperaldosteronismo Primário; Hipocaliemia.

\section{INTRODUÇÃO}

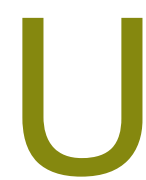

ma causa secundária de hipertensão é encontrada em 5 a $10 \%$ dos doentes submetidos a uma investigação etiológica da doença. $^{1}$

Estudos recentes revelam que a prevalência de hiperaldosteronismo primário pode ir de 5 a $13 \%$ entre os hipertensos..$^{2,3}$ Os doentes podem apresentar-se assintomáticos, com sintomas decorrentes da crise hipertensiva (cefaleia, tonturas, náuseas, vómitos, dispneia, dor torácica, défices neurológicos e síncope $)^{4}$ ou das complicações geradas pela hipocaliemia (poliúria, nictúria, cãibras musculares, fraqueza muscular excessiva, parestesias, tetania e até paralisias musculares). ${ }^{5}$ Do ponto de vista laboratorial, além da hipocaliemia, os resul-

*Médica Interna de Medicina Geral e Familiar Centro de Saúde de Corroios - Extensão de Corroios tados revelam concentrações elevadas de aldosterona plasmática e urinária, concentração de renina plasmática baixa, relação aldosterona/renina elevada e, por vezes, alcalose metabólica. ${ }^{6}$

Neste artigo, é apresentado o caso de um doente com hipertensão arterial primária controlada, que recorreu ao seu médico de família por crise hipertensiva, acompanhada por síncope recorrente e palpitações. A suspeita de uma causa secundária levou à investigação de hiperaldosteronismo primário, confirmando-se o diagnóstico.

Este caso clínico é ilustrativo da importância da continuidade de cuidados prestados pelo médico de família, permitindo o diagnóstico de um novo problema no contexto de patologia crónica previamente conhecida. Exemplifica que, na abordagem de um problema crónico, como é o caso da hipertensão arterial, num doente com polipatologia e polimedicado, como é comum 
nos doentes idosos, em que ocorre um agravamento da patologia, se verifica um aumento da complexidade da situação clínica, tornando imperativa uma avaliação global do doente. É, pois, fundamental que haja uma boa articulação entre os cuidados primários e os cuidados hospitalares, como se veio a mostrar neste caso.

\section{DESCRIÇÃO DO CASO}

HSG é um doente do sexo masculino, com 74 anos, de raça caucasiana, casado, com o $4^{\circ}$ ano de escolaridade, natural do Seixal e residente em Corroios. Foi operário fabril durante a maior parte da sua vida, tendo-se reformado aos 61 anos, por razões não relacionadas com o seu estado de saúde. O doente está inserido no seio de uma família nuclear, encontrando-se no estádio VIII do Ciclo de Vida de Duvall e na classe III de Graffar. No genograma, representado na Figura 1, há a referir 2 irmãos com hipertensão arterial, com início aos 47 anos, no irmão e aos 50 anos, na irmã.

O doente apresenta a seguinte lista de problemas:

- Hipertensão arterial desde há 20 anos,

- Dislipidemia mista desde há 11 anos;

- Estenose mitral diagnosticada há 10 anos e submetido a valvuloplastia com colocação de anel protésico mecânico, em posição mitral, há 9 anos;

- Fibrilhação auricular crónica há 8 anos, com aurícula esquerda dilatada.

Encontra-se medicado com os seguintes fármacos:

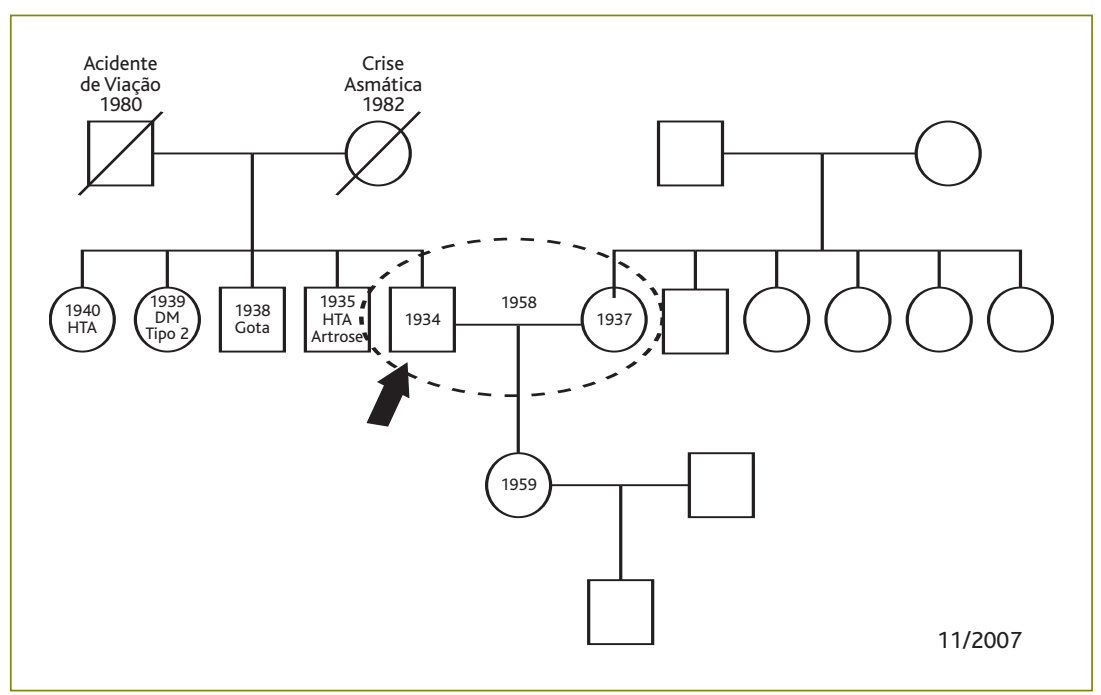

Figura 1. Genograma Familiar (11/2007)
- Hidroclorotiazida $50 \mathrm{mg}+$ Amilorida $5 \mathrm{mg} /$ dia p.o.;

- Pravastatina $20 \mathrm{mg} /$ dia p.o.;

- Varfarina $5 \mathrm{mg} 1 / 2 \mathrm{cp} /$ dia p.o.;

- Ticlopidina $250 \mathrm{mg} 2 \mathrm{cp} /$ dia p.o.;

- Digoxina 0,125 mg/dia p.o..

- O doente tem um Índice de Massa Corporal de 24,7. Tem por hábito consumir uma dieta com restrição de sal e de gorduras saturadas. Bebe, habitualmente, um copo de vinho tinto ao almoço e outro ao jantar. Não tem hábitos tabágicos e/ou toxicológicos. Não recorre a medicinas alternativas e não consome produtos de ervanária ou fármacos de venda livre. Pratica, regularmente, exercício físico aeróbio (marcha rápida de 30' diariamente).

Com o plano terapêutico instituído à data da última consulta com o médico de família (30/6/2006), a hipertensão arterial e a dislipidemia mista encontravam-se controladas, a fibrilhação auricular crónica tinha resposta ventricular controlada e o doente não apresentava qualquer complicação tromboembólica (decorrente da prótese valvular mecânica e da fibrilhação auricular) e/ou hemorrágica (decorrente da combinação do anticoagulante com o antiagregante plaquetário).

Na história deste doente não há relato de falta de adesão à terapêutica. Pelo contrário, é bastante interessado pelas doenças de que padece e com uma atitude sempre pró-activa, no sentido de melhorar ou, de, pelo menos, não agravar a sua situação clínica. Responsabiliza-se pelo cumprimento das medidas não farmacológicas e farmacológicas, aderindo facilmente ao plano terapêutico. $\mathrm{O}$ facto de ser consultado com regularidade pelo seu médico de família, permite uma monitorização apertada das doenças crónicas e da respectiva terapêutica.

\section{Consulta de Saúde de Adulto} $(28 / 9 / 2006)$

O doente recorreu ao seu médico de família por episódios sucessivos de «desmaio» (sic) no domicílio, no dia anterior, pela primeira vez. Referiu 3 episódios de perda súbita de conhecimento, que duraram cerca de 1 a 2 minutos, com recuperação es- 
pontânea e completa e que ocorreram em repouso. Referiu, ainda, palpitações com início abrupto, cerca de 30' antes dos episódios sincopais. Negou precordialgia, dispneia, sudorese, cefaleias e incontinência de esfíncteres, parestesias, fraqueza muscular, cãimbras, nictúria e poliúria. Negou, também, sintomas compatíveis com alterações da função tiroideia.

Ao exame objectivo apresentava-se em bom estado geral, eupneico, apirético, com mucosas coradas e hidratadas, evidenciando, no entanto, um estado de ligeira ansiedade pelo ocorrido no dia anterior. Não se observaram estrias cutâneas, acantose nigricans e/ou obesidade central. A pressão arterial em decúbito dorsal era 190/110 mmHg e em pé $170 / 100$ mmHg. O pulso radial era arrítmico, irregular, amplo e simétrico, frequência cardíaca $\pm 76 \mathrm{bpm}$. Auscultação cardíaca: arritmia, sopro sistólico grau II/VI na área mitral com irradiação axilar, sem atrito pericárdico. Auscultação pulmonar sem alterações. Abdómen: indolor, sem massas ou organomegalias, ruídos hidroaéreos presentes, sem sopros. Exame neurológico sem alterações, com fundoscopia aparentemente sem edema da papila, exsudados e/ou hemorragias.

Realizou-se teste colorimétrico da urina, que não revelou alterações e ECG, que revelou fibrilhação auricular e desvio esquerdo do eixo. Pediu-se hemograma completo, taxa de protrombina, função renal, ionograma sérico, urina tipo II, ecocardiograma e radiografia de tórax.

Medicou-se com captopril $25 \mathrm{mg}$ sl e diazepam $5 \mathrm{mg}$ po no centro de saúde. Ao fim de 2 horas, o doente não manifestava sintomas e tinha havido uma redução dos valores tensionais para 160/95 mmHg, medidos com o doente sentado. Manteve-se a medicação habitual e marcou-se consulta para o dia seguinte para reavaliação clínica e laboratorial.

\section{Consulta de Saúde de Adulto (29/9/2006)}

$\mathrm{O}$ doente referiu tonturas e ligeira fraqueza muscular, mostrando-se menos ansioso do que no dia anterior. $\mathrm{O}$ exame físico era, à excepção da pressão arterial, sobreponível: 170/100 mmHg em decúbito dorsal e 160/90 mmHg em pé.

O valor de $\mathrm{K}^{+}$plasmático era de $2,6 \mathrm{mmol} / \mathrm{L}$ (valor de referência: 3,5 - 5,0 mmol/L). Os restantes exames analíticos estavam dentro da normalidade. Os exames ima- giológicos ainda não tinham sido realizados.

O doente foi referenciado ao Serviço de Urgência (SU), com as seguintes hipóteses de diagnóstico: hipocaliemia secundária ao uso dos diuréticos ou hipocaliemia por hiperaldosteronismo primário agravado pelo uso dos diuréticos.

\section{Unidade de Internamento de Curta Duração (29/9 a 5/10/ 2006)}

Foi efectuada progressiva correcção da hipocaliemia, tendo o doente permanecido assintomático durante o período de internamento.

O ecocardiograma efectuado revelou hipertrofia do VE, com função sistólica global e segmentar mantida e insuficiência ligeira da válvula mitral, com anel protésico.

Suspendeu-se a hidroclorotiazida e a amilorida, passando a fazer lisinopril $20 \mathrm{mg} /$ dia e indapamida $1,5 \mathrm{mg} / \mathrm{dia}$, mantendo a restante terapêutica. Teve alta com indicação para continuar o seguimento na consulta do médico de família e com referência à consulta de endocrinologia, para investigação etiológica da hipocaliemia.

\section{Internamento no Serviço de Endocrinologia (18 a 20/10/2006)}

Por persistência da hipocaliemia $(3,0 \mathrm{mmol} / \mathrm{L})$, na consulta de endocrinologia, o doente foi internado, tendo permanecido assintomático.

Foi realizada tomografia computorizada abdominal superior, que mostrou um nódulo sólido de 2,0 x 1,5 cm na glândula supra-renal direita, compatível com adenoma (Figura 2).

Teve alta, com indicação para suspensão de espironolactona em ambulatório durante 4 a 6 semanas, para posterior estudo do hiperaldosteronismo primário, em contexto de internamento.

A correcção da hipocaliemia passou a fazer-se, apenas, com cloreto de potássio, $600 \mathrm{mg} /$ dia.

\section{Internamento no Serviço de Endocrinologia} (31/1 a 6/2/2007)

Após mais de 12 semanas de suspensão de espironolactona, procedeu-se, então, aos doseamentos de aldosterona plasmática e urinária e de renina plasmática (Quadro I). 




Figura 2. Tomografia computorizada mostrando nódulo na glândula supra-renal direita (seta branca), medindo $2,0 \mathrm{~cm} \times 1,5 \mathrm{~cm}$.

Durante o internamento o doente apresentou picos hipertensivos sob terapêutica com captopril $25 \mathrm{mg} 8 / 8 \mathrm{~h}$, pelo que se optou pela não realização da prova de infusão salina.

\section{Plano de Actuação}

Nesta altura, foi feita, pelo médico de família, uma avaliação global do doente, integrando a nova situação.

Calculou-se o risco cardiovascular global, segundo a classificação de Framingham, sendo este de $25 \%$.

Considerou-se necessário instituir medidas terapêuticas agressivas, não farmacológicas e farmacológicas (Quadros II e III). Delineou-se um plano terapêutico, negociando com o doente algumas das intervenções propostas, afim de se alcançarem os objectivos desejados.

Com as medidas instituídas e, adjuvando o cumprimento rigoroso do plano terapêutico por parte do doente, traçaram-se os seguintes objectivos:

- $\mathrm{PA}<135 / 85 \mathrm{mmHg}$ (valores obtidos por automedição),

- colesterol total $<200 \mathrm{mg} / \mathrm{dl}$ (vigilância anual),

- colesterol-LDL $<100 \mathrm{mg} / \mathrm{dl}$ (vigilância anual),

- colesterol-HDL $\geq 60 \mathrm{mg} / \mathrm{dl}$ (vigilância anual),

- potássio sérico 3,5-4,5 mmol/L (vigilância trimestral),

- INR 2,5 - 3,0 (valor alvo indicado na fibrilhação auricular crónica com prótese valvular mecânica, determinado mensalmente),

- fibrilhação auricular crónica com resposta ventricu-

\begin{tabular}{|c|c|c|}
\hline \multicolumn{3}{|c|}{$\begin{array}{l}\text { QUADRO I. Exames analíticos após suspensão de } \\
\text { espironolactona }\end{array}$} \\
\hline & VR $^{1}$ & $\begin{array}{l}\text { Valores } \\
\text { basais }\end{array}$ \\
\hline Aldosterona plasmática (ng/dl) & $4-31^{2}$ & 64,0 \\
\hline Aldosterona urinária ( $\mu \mathrm{g} / 24 \mathrm{~h})$ & $2-10$ & 70,4 \\
\hline Renina plasmática $(\mathrm{ng} / \mathrm{ml} / \mathrm{h})$ & $0,5-2,5^{2}$ & 0,2 \\
\hline $\mathrm{K}+$ plasmático (mmol/L) & $3,5-5,0$ & 2,8 \\
\hline
\end{tabular}

1. Valor de referência.

2. Valor com o doente em pé.

QUADRO II. Medidas não farmacológicas preconizadas no plano terapêutico

Medidas não farmacológicas

- Dieta rica em frutos, vegetais, alimentos ricos em potássio e com baixo teor de gorduras saturadas

- Redução da ingestão de sal

- Abstinência de consumo de álcool

- Actividade física aeróbia diária

- Automedição e registo da pressão arterial $2 x /$ semana

QUADRO III. Medidas farmacológicas preconizadas no plano terapêutico

Medidas farmacológicas

- Lisinopril 20 mg/dia p.o.

- Cloridrato de diltiazem $60 \mathrm{mg} 2 \mathrm{cp} /$ dia p.o.

- Espironolactona $100 \mathrm{mg} 1 / 2 \mathrm{cp} /$ dia p.o.

- Pravastatina $20 \mathrm{mg} / \mathrm{dia}$ p.o.

- Varfarina $5 \mathrm{mg} 1 / 2 \mathrm{cp} /$ dia p.o.

- Digoxina 0,125 mg/dia p.o.

lar controlada (com monitorização da digoxinemia, sendo os valores terapêuticos $0,5-2,2 \mathrm{ng} / \mathrm{ml}$ ).

Com a introdução da espironolactona no plano terapêutico houve necessidade de estar alerta para alguns aspectos inerentes ao fármaco:

- a determinação do potássio sérico fez-se semanalmente durante as primeiras 4 a 6 semanas para ajuste da dose.

- a determinação da digoxinemia passou a ser feita de 
3 em 3 meses, pois a espironolactona aumenta o tempo de semi-vida da digoxina, havendo necessidade de ajuste da sua dosagem;

- evitou-se a terapêutica concomitante com anti-inflamatórios não esteróides, que podem interferir com a eficácia da espironolactona;

- explicou-se ao doente alguns dos efeitos secundários da espironolactona, tais como a impotência, a diminuição da líbido e a ginecomastia.

Finalmente, dada a existência da prótese mitro aórtica mecânica, manteve-se, naturalmente, no plano terapêutico, a profilaxia antibiótica da endocardite bacteriana.

Para vigilância da prótese valvular mecânica, protocolou-se a realização de uma avaliação clínica pelo cardiologista, com pedido de ECG e ecocardiograma transtorácico, e sempre que aparecessem novos sintomas do foro cardiovascular.

O doente ficou a ser seguido trimestralmente na consulta de endocrinologia, para reavaliação imagiológica do nódulo da supra-renal direita e determinação dos valores de aldosterona e de renina plasmática e urinária e potássio e sódio plasmático e urinário.

Relativamente à consulta do médico de família, o doente manteve uma vigilância mensal, para determinação do INR e reavaliação clínica.

\section{Situação Actual}

O doente mantém-se assintomático, com pressão arterial de consultório de 160/90 mmHg, o que se atribui ao efeito da bata branca, já que os valores tensionais da automedição são inferiores a 135/85 mmHg. Os valores de potássio mantêm-se controlados. O nódulo da supra-renal direita mantém as mesmas dimensões. Os restantes objectivos propostos foram, igualmente, alcançados. Do ponto de vista cirúrgico, pondera-se o risco de morbimortalidade cardiovascular para a intervenção de ablação de nódulo da glândula supra-renal direita, atendendo à idade do doente e às patologias concomitantes.

\section{COMENTÁRIO}

Num doente com hipertensão arterial controlada, que apresenta, subitamente, uma crise hipertensiva, é necessário encetar medidas dirigidas à detecção de causas identificáveis e, eventualmente, curáveis, de hiper- tensão arterial.

Antes de iniciar a exploração de causas secundárias de hipertensão arterial, é necessário verificar a gravidade da crise hipertensiva e, para isso, a história e o exame físico são fundamentais.

A suspeita de lesão aguda de órgão alvo é crucial, pois permite diferenciar urgência hipertensiva (sem lesão aguda) de emergência hipertensiva (com lesão aguda), sendo o modo e a premência de actuação completamente díspares.

No doente idoso, como neste caso clínico, advém um aumento da complexidade na abordagem diagnóstica, não só pelas comorbilidades pré-existentes, como, também, pela polimedicação em curso. Na anamnese, procurou-se confirmar a adesão à terapêutica e investigar quanto ao consumo de fármacos de prescrição livre, que podem descontrolar os valores tensionais, como é o caso dos antitússicos, laxantes, expectorantes, descongestionantes ou AINE. Questionou-se o doente quanto ao uso de produtos de ervanária que contêm, frequentemente, alcaçuz, e que estão contra-indicados na hipertensão pelo seu efeito mineralocorticóide e que provocam hipocaliemia. ${ }^{7}$

Quanto ao exame físico, a determinação da pressão arterial fez-se com o doente sentado e em pé, para avaliar a depleção de volume. Concluiu-se não haver depleção de volume. O exame neurológico é importante para averiguar eventuais sinais focais, compatíveis com o diagnóstico de acidente vascular cerebral. Neste caso, a ausência de estado confusional, náuseas, vómitos e convulsões excluiu encefalopatia hipertensiva. Na fundoscopia não se observaram exsudados, hemorragias ou papiledema, o que excluiu, igualmente, encefalopatia hipertensiva. No exame cardiovascular não se auscultaram sopros de novo ao nível do précordio ou das carótidas e a avaliação dos pulsos periféricos não mostrou alterações, pelo que se excluiu patologia cardiovascular de aparecimento recente. Na auscultação pulmonar não foram audíveis fervores crepitantes na base de ambos os campos, o que excluiu edema pulmonar. $\mathrm{Na}$ avaliação laboratorial pediu-se: hemograma completo, uremia, creatinemia, ionograma sérico e urina tipo II, excluindo anemia e trombocitose, insuficiência renal, proteinúria, hematúria e cilindros tubulares e confirmando-se hipocaliemia. O electrocardiograma excluiu isquémia miocárdica. A radiografia do tórax per- 
mitiu medir o índice cardiotorácico e excluir derrame pleural. $^{8}$

Excluída a situação de emergência hipertensiva, cuja referência aos cuidados hospitalares deve ser imediata, o modo de actuação passou pela tranquilização do doente quanto à natureza benigna da situação clínica e pelo alívio dos sintomas. O objectivo do tratamento é reduzir a pressão arterial para valores seguros do ponto de vista hemodinâmico, preservando as funções: cardíaca, renal e cerebral. Qualquer redução brusca da pressão arterial está contra-indicada. A redução gradual da pressão arterial pode durar entre 24 a 48 horas. Do ponto de vista prático, deve-se baixar a pressão arterial média cerca de $25 \%$ do valor inicial ou reduzir a pressão arterial diastólica para valores entre $100 \mathrm{a}$ $110 \mathrm{mmHg}$. Neste caso, o objectivo foi atingido com diazepam $5 \mathrm{mg}$ e captopril $25 \mathrm{mg}$. Dentro dos fármacos de eleição para o tratamento da urgência hipertensiva (captopril, labetalol, minoxidil e clonidina), o captopril foi o escolhido, por ser o que apresenta um início de acção mais rápido e menos efeitos adversos. ${ }^{9}$

No contexto de urgência hipertensiva, o doente referiu a ocorrência de síncope recorrente precedida de palpitações. O diagnóstico diferencial da síncope é quase sempre um desafio para o clínico. No entanto, neste caso, as palpitações como sintoma prodrómico, a história pessoal de estenose mitral e a fibrilhação auricular crónica apontam para uma causa cardíaca. Habitualmente, o prognóstico é reservado, quer a causa primária seja estrutural ( $4 \%$ dos casos) ou funcional, nomeadamente disrrítmica ( $14 \%$ dos casos)..$^{10}$

Fármacos que interferem no ritmo cardíaco, como é o caso da digoxina, são uma potencial causa de síncope, e podem induzir arritmias, apesar dos níveis séricos estarem entre os valores terapêuticos. ${ }^{11}$

Os diuréticos tiazídicos, como é o caso da hidroclorotiazida, pelo seu mecanismo de acção, e, sobretudo, em doses elevadas, podem causar desequilíbrios electrolíticos, nomeadamente hipocaliemia. ${ }^{12}$ Por sua vez, a hipocaliemia potencia os efeitos tóxicos dos digitálicos. Do ponto de vista electrocardiográfico, observa-se um prolongamento da repolarização, ondas $\mathrm{T}$ de baixa-voltagem e ondas U proeminentes..$^{13}$ No entanto, as alterações electrocardiográficas podem não ser visualizáveis, tornando o diagnóstico mais difícil. Foi o que aconteceu neste caso, em que as alterações eram ines- pecíficas.

Todavia, tratando-se de um doente com fibrilhação auricular crónica e com prótese valvular, é necessário ter presente que existe um elevado risco de arritmias. Deste modo, na abordagem etiológica da síncope deveria ter-se pedido um ECG de Holter de 24 horas, de acordo com as Recomendações Internacionais. ${ }^{14} \mathrm{O}$ pedido deste exame está indicado em várias situações, incluindo a doença cardíaca conhecida ou a suspeita de síncope precedida por palpitações. ${ }^{11}$ As duas indicações enquadravam-se neste caso clínico.

Atendendo aos antecedentes de patologia valvular, o ecocardiograma foi pedido para exclusão/confirmação da causa cardíaca de síncope, de acordo com as Recomendações Internacionais. ${ }^{14}$

Para o despiste de arritmias como causa de síncope estão preconizados outros exames complementares, mas que só devem ser realizados quando existem achados clínicos específicos, tais como a prova de esforço e os estudos electrofisiológicos intracardíacos. Estes estudos estão indicados quando existe uma forte suspeita da causa de síncope ser a disrritmia ou quando existe doença cardíaca subjacente grave confirmada ou suspeita. ${ }^{15}$

Relativamente à terapêutica antitrombótica do doente com prótese valvular mecânica, existe uma discrepância entre as Recomendações Nacionais ${ }^{16} \mathrm{e}$ as Internacionais. ${ }^{17}$ As Recomendações Nacionais defendem uma prevenção do tromboembolismo apenas com anticoagulação oral com antivitamínicos K. ${ }^{16}$ As Recomendações Internacionais referem que a adição de um antiagregante plaquetário à anticoagulação oral está indicada, após se ponderar a razão risco/beneficio. ${ }^{17}$ Quem institui a terapêutica antitrombótica combinada deve ter isso em consideração. Neste caso, a terapêutica terá sido instituída por um cardiologista, que seguia o doente desde a intervenção da valvuloplastia e que optou pela combinação de anticoagulação oral e antiagregação plaquetária.

Segundo as normas de orientação clínica nacionais, no seguimento dos doentes hipertensos, uma vez iniciada a terapêutica anti-hipertensora, a creatinemia e a caliemia deverão ser avaliadas, pelo menos, uma a duas vezes por ano. Nos idosos são frequentes sintomas de hipoperfusão cerebral, associado a desequilíbrios electrolíticos, sobretudo se houver uso de diuréticos. ${ }^{18}$ 
Com a introdução da espironolactona houve necessidade de determinar a caliemia semanalmente durante as primeiras 4 a 6 semanas para ajuste da dose, passando, depois, a avaliar-se bianualmente.

Neste caso, a hipocaliemia foi detectada através do pedido do ionograma sérico de urgência. Pelo uso de altas doses de hidroclorotiazida supôs-se ser uma hipocaliemia secundária ao diurético. Atendendo à suspeita de uma causa secundária, pela ocorrência da urgência hipertensiva, e à caliemia inferior a $3,1 \mathrm{mmol} / \mathrm{L}$, colocou-se a hipótese diagnóstica de hiperaldosteronismo primário agravado pelo uso prolongado de hidroclorotiazida.

Quando o doente teve alta da Unidade de Internamento de Curta Duração foi-lhe feita a substituição da associação de diuréticos (hidroclorotiazida + amilorida) por indapamida, outro diurético potencialmente hipocalemiante. Esta decisão, cuja causa se desconhece, contribuiu para a persistência da hipocaliemia, que motivou o internamento no Serviço de Endocrinologia.

O hiperaldosteronismo primário é caracterizado por retenção de sódio, hipertensão arterial, alcalose metabólica, níveis elevados de potássio urinário, supressão da actividade plasmática de renina ou níveis baixos da própria enzima e aumento da produção de aldosterona. A hipocaliemia é encontrada nas formas mais graves da doença, mas alguns autores sugerem que concentrações normais de potássio podem ser a regra. Embora a retenção de sódio seja o mecanismo inicial na fisiopatologia da hipertensão por excesso de mineralocorticóides, o aumento da resistência periférica total prevalece como principal alteração fisiopatológica a longo prazo. ${ }^{19}$

As duas principais etiologias são o adenoma produtor de aldosterona e a hiperplasia supra-renal ou hiperaldosteronismo idiopático, sendo a primeira mais prevalente $(75 \%) .^{5}$

Para o estudo do hiperaldosteronismo primário é fundamental a suspensão de diuréticos poupadores de potássio, nomeadamente a espironolactona, porque estes irão normalizar os electrólitos e os níveis de aldosterona e de renina. ${ }^{8}$

No caso clínico, os exames analíticos mostraram potássio plasmático baixo, sugerindo excesso de mineralocorticóide, aldosterona plasmática e urinária elevadas e renina plasmática diminuída, indicando provável secreção autónoma de aldosterona. Por sua vez, esta última deve ser comprovada pela ausência de resposta aos testes de supressão. Dentre os testes mais utilizados está a prova de infusão salina, que consiste na infusão endovenosa de 2,5 litros de solução fisiológica ( $\mathrm{NaCl}$ a $0,9 \%$ ) durante 2 horas, com determinação da concentração de aldosterona plasmática antes e no final da infusão. ${ }^{5}$ Contudo, esta prova não é desprovida de riscos. Pode existir uma sobrecarga volémica e a indução de insuficiência cardíaca. Nos doentes com risco de descompensação da patologia cardíaca prévia, caso se pondere a realização da prova, a infusão deve ser feita durante 4 horas. No caso clínico, se a prova se tivesse realizado, os resultados seriam a manutenção dos níveis elevados de aldosterona plasmática antes e no final da infusão.

Os resultados analíticos foram corroborados pelo exame imagiológico que mostrou um nódulo na supra-renal direita, compatível com adenoma.

O adenoma produtor de aldosterona é uma das poucas causas potencialmente curáveis de hipertensão arterial. Estes tumores são normalmente pequenos (menos de $2 \mathrm{~cm}$ de diâmetro) e benignos. ${ }^{20} \mathrm{~A}$ remoção cirúrgica resulta na normalização da hipertensão arterial em 65 a 70\% dos casos e da hipocaliemia em 100\% dos casos. A adrenalectomia não está indicada em doentes idosos, para os quais é preferível o tratamento com anti-hipertensores, nomeadamente a espironolactona. ${ }^{19}$

O médico de família, como agente prestador de cuidados primários, exerce um papel fundamental na articulação de cuidados a diferentes níveis e tende a abordar o doente de uma forma holística.

Relativamente a este caso, o médico de família exerce um papel imprescindível na vigilância laboratorial, em complemento com a dos outros especialistas, e na recolha de informações sobre exames ou intervenções relevantes efectuados ao nível dos cuidados hospitalares. Tem um papel importante na preparação do doente e aconselhamento sobre aspectos eventualmente menos valorizados, como os psicológicos, na preparação para uma eventual cirurgia e impacto que daí advém para a família.

No futuro, o médico de família irá efectuar um estudo dirigido a esta patologia, no que diz respeito ao prognóstico perante a não intervenção cirúrgica, e eventuais medidas terapêuticas a adoptar, atendendo à ava- 
liação global do doente, dados que poderão beneficiálo e servir para melhor o educar.

Reflectindo sobre o caso clínico apresentado, é importante reter:

A grande maioria dos doentes com hipertensão arterial apresenta a forma primária. Contudo, durante a evolução da doença podem surgir formas secundárias, potencialmente curáveis.

A suspeita de hipertensão arterial secundária pressupõe o conhecimento das possíveis etiologias e respectivas manifestações clínicas. O hiperaldosteronismo primário é uma das causas secundárias mais prevalentes.

Os valores tensionais anormalmente elevados e a crise hipertensiva levantam a suspeita de hipertensão arterial secundária e a hipocaliemia acentuada levanta a suspeita de hiperaldosteronismo primário.

A história clínica detalhada e o exame físico rigoroso, associados ao pedido descriminado de exames complementares de diagnóstico, são mandatórios na investigação etiológica.

\section{REFERÊNCIAS BIBLIOGRÁFICAS}

1. Onusko E. Diagnosing secondary hypertension. Am Fam Physician 2003 Jan 1; 67 (1): 67-74.

2. Nishikawa T, Saito J, Omura M. Prevalence of primary aldosteronism: should we screen for primary aldosteronism before treating hypertensive patients with medication? Endocr J 2007 Aug; 54 (4): 487-95.

3. Young WF Jr. Minireview: primary aldosteronism - changing concepts in diagnosis and treatment. Endocrinology 2003 Jun; 144 (6): 2208-13 .

4. Martin JF, Higashiama E, Garcia E, Luizon MR, Cipullo JP. Perfil de crise hipertensiva: prevalência e apresentação clínica. Arq Bras Cardiol 2004 Aug; 83 (2): 125-36.

5. Kater CE. Hiperaldosteronismo primário. Arq Bras Endocrinol Metabol 2002 Fev; 46 (1): 106-15.

6. Plouin PF, Jeunemaitre $X$. Would wider screening for primary aldosteronism give any health benefits? Eur J Endocrinol 2004 Sep; 151 (3): 305-8.

7. Mendes A, Simón A. Interacções de produtos à base de plantas com medicamentos - II, Ficha Técnica N²51. Revista da Ordem dos Farmacêuticos $2003 \mathrm{Jan} / \mathrm{Fev}$; 51: 65-6.

8. Aggarwal M, Khan IA. Hypertensive crisis: hypertensive emergencies and urgencies. Cardiol Clin 2006 Feb; 24 (1):135-46.
9. Fenves $A Z$, Ram CV. Drug treatment of hypertensive urgencies and emergencies. Semin Nephrol 2005 Jul; 25 (4): 272-80.

10. Marques F, Fonseca C, Sarmento P, Sousa I, Jorge A, Santos JM, et al. Síncope recorrente - um desafio disgnóstico. Rev Port Cardiol 2006 Abr; 25 (4): 431-41.

11. Miller TH, Kruse JE. Evaluation of syncope. Am Fam Physician 2005 Oct 15; 72 (8): 1492-500.

12. Instituto Nacional da Farmácia e do Medicamento (INFARMED). Prontuário Terapêutico - 6. Lisboa: INFARMED; 2006.

13. Lipman BC, Cascio T. ECG: avaliação e interpretação. Loures: Lusociência - Edições Técnicas e Científicas; 2001.

14. Brignole M, Alboni P, Benditt DG, Bergfeldt L, Blanc JJ, Thomsen PE, et al. Guidelines on management (diagnosis and treatment) of syncope - update 2004. Europace 2004 Nov; 6 (6): 467-537.

15. Branch WT, Alexander RW, Schlant RC, Hurst JW. Cardiology in Primary Care. New York: McGraw-Hill; 2001.

16. Gomes RS, Aguiar C, Ferro J, Henriques IL, Crespo F, Carvalho HC, et al. Recomendações sobre terapêutica antitrombótica da fibrilhação auricular. Lisboa: Alto Comissariado da Saúde; 2008.

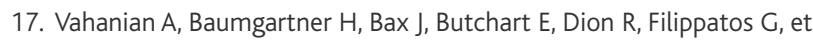
al. Guidelines on the management of valvular heart disease: The Task Force on the Management of Valvular Heart Disease of the European society of Cardiology. Eur Heart J 2007 Jan; 28 (2): 230-68.

18. Direcção Geral de Saúde (DGS). Diagnóstico, tratamento e controlo da hipertensão arterial. Lisboa: DGS; 2004.

19. Panarotto D, Carli CS, Cavagnoli M, Macedo CL, Zanettini LA, Biasin VF. Hiperaldosteronismo primário causado por adenoma adrenal produtor de aldosterona. Rev AMRIGS 2004; 48 (1): 32-6.

20. Martin JF, Vicente AR, Cury PM, Dib JA, Cipullo JP. Adenoma de Conn: uma causa de hipertensão e hipocaliemia. Arq Bras Cardiol 2004 Jul; 83 (1): 83-6.

\section{ENDEREÇO PARA CORRESPONDÊNCIA}

Fátima Carvalho Matos

E-mail:matos_fatima@clix.pt

\section{AGRADECIMENTOS}

Agradeço o entusiasmo da minha orientadora de internato, Dra. Gabriela Fernandes, bem como a sua disponibilidade para comentar e corrigir este artigo.

Recebido em 03/06/08

Aceite para publicação em 31/12/08 


\section{ABSTRACT}

Context: The vast majority of patients with hypertension present the primary form. However, secondary forms may arise in the course of the disease, the most prevalent being the primary hyperaldosteronism. Between the two main causes are the aldosterone-producing adenoma and adrenal hyperplasia, the first being most common.

Case description: 74 years old male patient, hypertensive with chronic atrial fibrillation controlled with diuretics and anti-arrhythmic drug, subjected to valvuloplasty for mitral stenosis, is in the query by elevated blood pressure levels associated with syncope and palpitations. In laboratory evaluation was detected hypokalemia, which raised the suspicion of adverse effect of diuretic and / or hyperaldosteronism. The final diagnosis was confirmed after further studies, which indicated high plasmatic and urinary aldosterone and low plasmatic renin. The computed tomography detected a solid nodule in right adrenal, consistent with adenoma. The patient was referred for consultation of Endocrinology, medicated with spironolactone. Awaiting possible unilateral adrenalectomy.

Comment: The case shows that during the development of hypertension, secondary potentially curable cases may occur, for which the family doctor must be alert. A detailed medical history and a thorough physical examination, associated with the application of the diagnostic tests, make it possible to ascertain the cause of secondary hypertension. This case is illustrative of the importance that is the continuity of care provided by family doctors and of a good coordination between primary care and hospital care.

Keywords: Secondary Hypertension; Primary Aldosteronism; Hypokalemia. 